\title{
Non-positive upper bounds on expectations of small order statistics from DDA and DFRA populations
}

\author{
Tomasz Rychlik
}

Received: 24 November 2012 / Published online: 3 August 2013

(C) The Author(s) 2013. This article is published with open access at Springerlink.com

\begin{abstract}
Rychlik [Appl Math (Warsaw) 29:15-32, 2002] presented positive sharp upper bounds on the expectations of order statistics with sufficiently large ranks, based on i.i.d. samples from the decreasing density and failure rate populations (DDA and DFRA, for short). They were expressed in terms of the population mean and standard deviation. Here we provide respective non-positive upper tight evaluations for expected small order statistics centered about the population mean, measured in various scale units.
\end{abstract}

Keywords Order statistic - Decreasing density on the average .

Decreasing failure rate on the average $\cdot$ Sharp bound

\section{Introduction}

We assume that $X_{1}, \ldots, X_{n}$ are i.i.d. random variables, and $X_{1: n} \leq \cdots \leq X_{n: n}$ stand for the respective order statistics. We denote by $F, \mu=\mathbb{E} X_{1}$, and $\sigma_{p}^{p}=\mathbb{E}\left|x_{1}-\mu\right|^{p}$ for some $p \geq 1$, the respective parent distribution function, mean, and $p$ th absolute central moment. Writing $\sigma_{p}^{p}$ or $\sigma_{p}=\left(\sigma_{p}^{p}\right)^{1 / 2}$ below, we tacitly assume that they are positive and finite. Let

$$
F^{-1}(x)=\sup \{u: F(u) \leq x\}, \quad 0 \leq x<1,
$$

be the upper quantile function of $F$. We also assume that $a_{F}=F^{-1}(0)>-\infty$, i.e., $F$ has a support bounded below, and

T. Rychlik (凶)

Institute of Mathematics, Polish Academy of Sciences, Chopina 12, 87100 Toruń, Poland

e-mail: trychlik@impan.pl 


$$
\frac{F^{-1}(x)-a_{F}}{x}, \quad 0<x<1,
$$

is a non-decreasing function. An equivalent condition is that $F(x) /\left(x-a_{F}\right), x>a_{F}$, is non-increasing which means that $F$ has a possible jump at $a_{F}$, and a density function $f$ such that $\frac{1}{x-a_{F}}\left[F\left(a_{F}\right)+\int_{a_{F}}^{x} f(t) d t\right]$ does not increase for $x>a_{F}$. If $F\left(a_{F}\right)=0$, one could say that the density function is non-increasing on the average. We extend the definition to the distributions with atoms described above in order to make the family of decreasing density on the average distributions (DDA) closed.

Moreover, we consider a narrower family of decreasing failure rate on the average distribution functions (DFRA) for which functions

$$
\frac{F^{-1}\left(1-e^{-x}\right)-a_{F}}{x}=\frac{F^{-1}\left(1-e^{-x}\right)-a_{F}}{1-e^{-x}} \frac{1-e^{-x}}{x}, \quad x>0,
$$

are non-decreasing. Note that the latter fraction of the RHS is decreasing, and so (2) is indeed a stronger condition than (1). In other words, for the DFRA distributions the function

$$
\frac{-\ln (1-F(x))}{x-a_{F}}=\frac{-\ln \left(1-F\left(a_{F}\right)\right)+\int_{a_{F}}^{x} \frac{f(t)}{1-F(t)} d t}{x-a_{F}}, \quad x>a_{F},
$$

does not increase. Usually the DFRA family is treated as a subfamily of life distributions with $a_{F}=0$. Here we drop this restrictive assumption.

We aim at providing precise upper evaluations on $\mathbb{E}_{F} X_{j: n}-\mu$, expressed in various scale units $\sigma_{p}, p \geq 1$, when the parent distributions come from the DDA and DFRA families. It is intuitively obvious that these bounds can be positive for large $j$ (with respect to $n$ ), and negative for small ones. Rychlik (2002) presented conditions on the pairs $(j, n)$ such that $\mathbb{E}_{F} X_{j: n} \leq \mu$ when the marginal distribution is either DDA or DFRA. We recall them below. Since relations $\mathbb{E}_{F} X_{1: n} \leq \mu \leq \mathbb{E}_{F} X_{n: n}$ are valid for arbitrary distributions of $X_{i}$ 's, we focus now on $2 \leq j \leq n-1$. Formula

$$
f_{j: n}(x)=n\left(\begin{array}{c}
n-1 \\
j-1
\end{array}\right) x^{j-1}(1-x)^{n-j}, \quad 0<x<1,
$$

defines the density function of the $j$ th order statistic based on $n$ i.i.d. standard uniform random variables. If $2 \leq j \leq n-1$, it continuously increases from $f_{j: n}(0)=0$ to $f_{j: n}\left(\frac{j-1}{n-1}\right)>1$, and ultimately decreases to $f_{j: n}(1)=0$. Let $0<a_{j: n}<\frac{j-1}{n-1}<$ $b_{j: n}<1$ denote the smaller and greater arguments at which $f_{j: n}$ amounts to 1 . Let

$$
\begin{aligned}
& F_{j: n}(x)=\sum_{i=j}^{n}\left(\begin{array}{l}
n \\
i
\end{array}\right) x^{i}(1-x)^{n-i}, \\
& \bar{F}_{j: n}(x)=\sum_{i=0}^{j-1}\left(\begin{array}{l}
n \\
i
\end{array}\right) x^{i}(1-x)^{n-i}, \quad 0<x<1,
\end{aligned}
$$


denote the distribution and survival functions of of the $j$ th order statistic from the standard uniform sample. We also define

$$
\begin{aligned}
K_{j: n}(x) & =\frac{j}{n+1} \bar{F}_{j+1: n+1}(x)-\frac{1-x^{2}}{2} \\
L_{j: n}(x) & =\sum_{i=1}^{j} \frac{\bar{F}_{i: n}(x)}{n+1-i}-\bar{F}_{j: n}(x) \ln (1-x)-(1-x)[1-\ln (1-x)] .
\end{aligned}
$$

Proposition 1 (Rychlik 2002, Propositions 1 and 2) Let random variables $X_{1}, \ldots, X_{n}$ be i.i.d. with a common distribution function $F$ and mean $\mu$. If either

(a) $F$ is DDA and either $j=1$ or $K_{j: n}\left(a_{j: n}\right) \leq 0$ for $j \geq 2$, or

(b) $F$ is DFRA and either $j=1$ or $L_{j: n}\left(a_{j: n}\right) \leq 0$ for $j \geq 2$, then $\mathbb{E}_{F} X_{j: n} \leq \mu$.

Rychlik (2002) established positive sharp upper bounds on $\mathbb{E}_{F}\left(X_{j: n}-\mu\right) / \sigma_{2}$ when either $F$ is DDA and $K_{j: n}\left(a_{j: n}\right)>0$ or $F$ is DFRA and $L_{j: n}\left(a_{j: n}\right)>0$. Here we focus on calculating sharp non-positive bounds on $\mathbb{E}_{F}\left(X_{j: n}-\mu\right) / \sigma_{p}$ under assumptions (a) and (b) of Proposition 1. The DDA and DFRA families of distributions are treated in Sects. 2 and 3, respectively. We show that in both the cases these bounds amount trivially to 0 when $p>1$. For $p=1$, we describe strictly negative evaluations. We also determine (sequences of) distributions which attain the bounds (possibly in the limit). We conclude Sects. 2 and 3 with presenting numerical values of negative sharp bounds on $\mathbb{E}_{F}\left(X_{j: n}-\mu\right) / \sigma_{1}$ in the DDA and DFRA cases, respectively, for the samples of sizes $n=10,20$ and 30 .

We notice that the families of DDA and DFRA distributions can be defined in a coherent way by use of the star order of distributions, introduced by Barlow et al. (1969) (see also Shaked and Shantikumar 2007 for a comprehensive study). We say that $F$ succeeds $G$ in the order iff the composition $F^{-1} \circ G$ is starshaped, i.e. function $\left[F^{-1}(G(x))-F^{-1}\left(G\left(a_{G}\right)\right)\right] /\left(x-a_{G}\right)$ is non-decreasing. The DDA and DFRA distribution functions are the ones that succeed the uniform and exponential distributions in the star ordering. Our results can be extended onto the families of distributions succeeding a general fixed distribution in the star order.

The expectations of order statistics coming from general i.i.d. samples were evaluated in terms of the population mean and standard deviation by Moriguti (1953). Analytic formulae for respective bounds on the sample maxima were determined by Gumbel (1954) and Hartley and David (1954). Analogous results for more general scale units, generated by central absolute moments of various orders were determined by Arnold (1985) for the sample maxima, and Rychlik (1998) for the other order statistics. The first optimal evaluations of the expectations of order statistics from the decreasing density and failure rate populations (DD and DFR, respectively) are due to Gajek and Rychlik (1998). They were represented in the scale units connected with the second raw moments of the parent distribution. Positive sharp mean-standard deviation estimates of the expected order statistics and spacings from the DD and DFR families were determined by Danielak (2003) and Danielak and Rychlik (2004), respectively. 
Barlow and Proschan (1966) and Barlow et al. (1969) derived some inequalities for $L$-statistics implied by the star ordering of the distributions of observations [see also Arnold nad Balakrishnan (1989, Section 3.4)]. Rychlik (2002) determined positive tight upper mean-standard deviation bounds on the expectations of order statistics with sufficiently large ranks, coming from DDA and DFRA populations. Similar results for the spacings are due to Danielak and Rychlik (2003). Bieniek $(2006,2008)$ presented analogous evaluations for the generalized order statistics based on the DFR, DFRA, DD and DDA populations.

Tight positive lower (and negative upper) bounds on the expectations of positive (negative) $L$-statistics with general parent distributions, expressed in various scale units, were obtained by Goroncy (2009). Negative upper estimates for order statistics less than the sample median, based on symmetric and symmetric unimodal distributions can be found in Rychlik (2009a). Rychlik (2009b,c) provided analogous results for small order statistics coming from DD and DFR populations, respectively. Negative sharp upper evaluations for the expectations of generalized order statistics coming from arbitrary populations were studied in Goroncy (2013).

\section{DDA distributions}

Here we collect the assumptions valid throughout this section.

Assumptions $D D A(p)$ : Random variables $X_{1}, \ldots, X_{n}$ are i.i.d. with a common distribution function $F$ which is DDA, i.e. it has a finite left end-point $a_{F}$ of the support, and function $F(x) /\left(x-a_{F}\right), x>a_{F}$, is non-increasing. $X_{1}$ has a finite expectation $\mathbb{E}_{F} X_{1}=\mu$ and positive finite $p$ th central absolute moment $0<\sigma_{p}^{p}=$ $\mathbb{E}_{F}\left|X_{1}-\mu\right|^{p}<\infty$ for some $1 \leq p<\infty$. We also assume that either $j=1$ or $2 \leq j \leq n-1$ is such that $K_{j: n}\left(a_{j: n}\right) \leq 0$ (cf. Proposition $\left.1 a\right)$, where function $K_{j: n}$ is defined in (4), and $a_{j: n}$ is the smaller of two roots of equation $f_{j: n}(x)=1$ in $(0,1)$ [see (3)]. We put

$$
M_{p}(\alpha)=\left[\frac{2(p+1) \alpha(1-\alpha)^{p}+\left(1+\alpha^{2}\right)^{p+1}-\left(\alpha^{2}+2 \alpha-1\right)\left|\alpha^{2}+2 \alpha-1\right|^{p}}{2^{p+1}(p+1)}\right]^{1 / p}
$$

for $0 \leq \alpha<1$ and $p \geq 1$. In particular, for $p=1$ we have

$$
\begin{aligned}
M_{1}(\alpha) & =\frac{1}{8}\left[4 \alpha\left(1-\alpha^{2}\right)+\left(1+\alpha^{2}\right)^{2}-\operatorname{sgn}\left(\alpha^{2}+2 \alpha-1\right)\left(\alpha^{2}+2 \alpha-1\right)^{2}\right] \\
& = \begin{cases}\frac{\left(\alpha^{2}+1\right)^{2}}{4}, & 0 \leq \alpha \leq \sqrt{2}-1, \\
\alpha\left(1-\alpha^{2}\right), & \sqrt{2}-1 \leq \alpha<1 .\end{cases}
\end{aligned}
$$

Proposition 2 (Case $p>1$ ) If the above assumptions DDA $(p)$ hold for some $p>1$ and $1 \leq j<n$, then the bound

$$
\frac{\mathbb{E}_{F} X_{j: n}-\mu}{\sigma_{p}} \leq 0
$$


is sharp, and it is attained in the limit by the family of mixtures of atoms at $\mu-$ $\frac{\sigma_{p}}{2 M_{p}(\alpha)}\left(1-\alpha^{2}\right)$ and uniform distributions on the intervals $\left[\mu+\frac{\sigma_{p}}{2 M_{p}(\alpha)}\left(2 \alpha-1+\alpha^{2}\right)\right.$, $\left.\mu+\frac{\sigma_{p}}{2 M_{p}(\alpha)}\left(1+\alpha^{2}\right)\right]$ with weights $\alpha$ and $1-\alpha$, respectively, as $\alpha \nearrow 1$.

Proof Inequality (8) is obvious in view of Proposition 1. It merely suffices to check that the distributions defined in the latter claim are DDA, satisfy the moment conditions, and attain the zero bound in the limit. Note that the respective quantile functions satisfy

$$
\frac{F_{\alpha}^{-1}(x)-\mu}{\sigma_{p}}=\frac{x \mathbf{1}_{[\alpha, 1)}(x)-\frac{1-\alpha^{2}}{2}}{M_{p}(\alpha)}, \quad 0 \leq x<1
$$

Therefore

$$
\frac{F_{\alpha}^{-1}(x)-\mu+\frac{\sigma_{p}}{2 M_{p}(\alpha)}\left(1-\alpha^{2}\right)}{x}=\frac{\sigma_{p}}{M_{p}(\alpha)} \mathbf{1}_{[\alpha, 1)}(x), \quad 0 \leq x<1
$$

are non-decreasing, and so $F_{\alpha}, 0 \leq \alpha<1$, are DDA. Since

$$
\int_{0}^{1}\left[F_{\alpha}^{-1}(x)-\mu\right] d x=\frac{\sigma_{p}}{M_{p}(\alpha)}\left[\int_{0}^{1} x \mathbf{1}_{[\alpha, 1)}(x) d x-\frac{1-\alpha^{2}}{2}\right]=0,
$$

we have $\mathbb{E}_{\alpha} X_{1}=\mu$. Now we calculate

$$
\begin{aligned}
& \int_{0}^{1}\left|x \mathbf{1}_{[\alpha, 1)}(x)-\frac{1-\alpha^{2}}{2}\right|^{p} d x=\int_{0}^{\alpha}\left(\frac{1-\alpha^{2}}{2}\right)^{p} d x \\
& \quad+ \begin{cases}\int_{\alpha}^{\left(1-\alpha^{2}\right) / 2}\left(\frac{1-\alpha^{2}}{2}-x\right)^{p} d x+\int_{\left(1-\alpha^{2}\right) / 2}^{1}\left(x-\frac{1-\alpha^{2}}{2}\right)^{p} d x, & 0 \leq \alpha \leq \sqrt{2}-1, \\
\int_{\alpha}^{1}\left(x-\frac{1-\alpha^{2}}{2}\right)^{p} d x, & \sqrt{2}-1 \leq \alpha<1,\end{cases} \\
& =\frac{\left(1-\alpha^{2}\right)^{p} \alpha}{2^{p}}+ \begin{cases}\frac{\left(1-\alpha^{2}-2 \alpha\right)^{p+1}+\left(1+\alpha^{2}\right)^{p+1}}{2^{p+1}(p+1)}, & 0 \leq \alpha \leq \sqrt{2}-1, \\
\frac{\left(1+\alpha^{2}\right)^{p+1}-\left(1-\alpha^{2}-2 \alpha\right)^{p+1}}{2^{p+1}(p+1)}, & \sqrt{2}-1 \leq \alpha<1,\end{cases}
\end{aligned}
$$

Therefore

$\mathbb{E}_{\alpha}\left|X_{1}-\mu\right|^{p}=\int_{0}^{1}\left|F_{\alpha}^{-1}(x)-\mu\right|^{p} d x=\frac{\sigma_{p}^{p}}{M_{p}^{p}(\alpha)} \int_{0}^{1}\left|x \mathbf{1}_{[\alpha, 1)}(x)-\frac{1-\alpha^{2}}{2}\right|^{p} d x=\sigma_{p}^{p}$, 
as claimed. Furthermore

$$
\begin{aligned}
\frac{\mathbb{E}_{\alpha} X_{j: n}-\mu}{\sigma_{p}} & =\int_{0}^{1} \frac{F_{\alpha}^{-1}(x)-\mu}{\sigma_{p}} f_{j: n}(x) d x \\
& =\int_{0}^{1} \frac{x \mathbf{1}_{[\alpha, 1)}(x)-\frac{1-\alpha^{2}}{2}}{M_{p}(\alpha)} f_{j: n}(x) d x \\
& =\frac{1}{M_{p}(\alpha)}\left[\int_{\alpha}^{1} x f_{j: n}(x) d x-\frac{1-\alpha^{2}}{2}\right] \\
& =\frac{1}{M_{p}(\alpha)}\left[\frac{j}{n+1} \int_{\alpha}^{1} f_{j+1: n+1}(x) d x-\frac{1-\alpha^{2}}{2}\right]=\frac{K_{j: n}(\alpha)}{M_{p}(\alpha)} .
\end{aligned}
$$

[cf (4) and (6)]. Note that

$$
\begin{aligned}
K_{j: n}(\alpha) & =\frac{j}{n+1} \sum_{i=0}^{j}\left(\begin{array}{c}
n+1 \\
i
\end{array}\right) \alpha^{i}(1-\alpha)^{n+1-i}-\frac{1-\alpha^{2}}{2} \\
& =(1-\alpha)\left[\frac{j}{n+1} \sum_{i=0}^{j}\left(\begin{array}{c}
n+1 \\
i
\end{array}\right) \alpha^{i}(1-\alpha)^{n-i}-\frac{1+\alpha}{2}\right],
\end{aligned}
$$

and the expression in the square brackets tends to -1 , as $\alpha \nearrow 1$. Indeed, the sum does not converge there only for $j=n$. However, this case is excluded from our study because $\mathbb{E}_{F} X_{n: n} \geq \mu$. So we have $K_{j: n}(\alpha) \sim-(1-\alpha)$ as $\alpha \nearrow 1$.

For $\alpha \geq \sqrt{2}-1$, we have

$$
M_{p}^{p}(\alpha)=\frac{2(p+1) \alpha(1-\alpha)^{p}+\left(1+\alpha^{2}\right)^{p+1}-\left(\alpha^{2}+2 \alpha-1\right)^{p+1}}{2^{p+1}(p+1)} .
$$

Applying the Taylor expansions, we obtain

$$
\begin{aligned}
\left(1+\alpha^{2}\right)^{p+1} & =2^{p+1}-(p+1) 2^{p+1}(1-\alpha)+\mathcal{O}\left((1-\alpha)^{2}\right), \\
\left(\alpha^{2}+2 \alpha-1\right)^{p+1} & =2^{p+1}-(p+1) 2^{p+2}(1-\alpha)+\mathcal{O}\left((1-\alpha)^{2}\right) .
\end{aligned}
$$

Hence $M_{p}^{p}(\alpha)=(1-\alpha)+\mathcal{O}\left((1-\alpha)^{\min \{2, p\}}\right)$, and so

$$
\frac{K_{j: n}(\alpha)}{M_{p}(\alpha)} \sim-(1-\alpha)^{1-1 / p} \rightarrow 0 \text { as } \alpha \nearrow 1
$$

which is the desired statement. 
Proposition 3 (Case $p=1$ ) If assumptions $D D A(p)$ are satisfied for $p=1$ and $1 \leq j<n$, then

$$
\frac{\mathbb{E}_{F} X_{j: n}-\mu}{\sigma_{1}} \leq \sup _{0 \leq \alpha<1} \frac{K_{j: n}(\alpha)}{M_{1}(\alpha)},
$$

[see (4) and (7)]. If the supremum in (9) is attained at some $0 \leq \alpha=\alpha_{j: n}<1$, then the bound is attained by the mixture of the pole at $\mu-\frac{\sigma_{1}}{2 M_{1}(\alpha)}\left(1-\alpha^{2}\right)$ and the uniform distribution on $\left[\mu+\frac{\sigma_{1}}{2 M_{1}(\alpha)}\left(2 \alpha-1+\alpha^{2}\right), \mu+\frac{\sigma_{1}}{2 M_{1}(\alpha)}\left(1+\alpha^{2}\right)\right]$ with weights $\alpha$ and $1-\alpha$, respectively. If

$$
\sup _{0 \leq \alpha<1} \frac{K_{j: n}(\alpha)}{M_{1}(\alpha)}=\lim _{\alpha \nearrow 1} \frac{K_{j: n}(\alpha)}{M_{1}(\alpha)}=-\frac{1}{2},
$$

then the bound is attained in the limit by the sequences of mixtures described above as $\alpha \nearrow 1$.

Proof By Proposition 1, we have

$$
\sup _{F \in D D A(1)} \frac{\mathbb{E}_{F} X_{j: n}-\mu}{\sigma_{1}} \leq 0,
$$

where $D D A(1)$ denotes the family of non-degenerate $D D A$ distributions with a finite first moment. Below we prove the implications

$$
\begin{gathered}
K_{j: n}\left(a_{j: n}\right)=0 \Rightarrow \sup _{F \in D D A(1)} \frac{\mathbb{E}_{F} X_{j: n}-\mu}{\sigma_{1}}=0, \\
\sup _{F \in D D A(1)} \frac{\mathbb{E}_{F} X_{j: n}-\mu}{\sigma_{1}}<0 \Rightarrow \text { either } j=1 \text { or } K_{j: n}\left(a_{j: n}\right)<0,
\end{gathered}
$$

which together imply that both (10) and (11) are equivalence relations.

We first observe that the mixtures defined in the statements of the proposition are DDA, and satisfy both the moment conditions. It suffices to recall respective arguments of the proof of Proposition 2 for specific $p=1$. We also get

$$
\frac{\mathbb{E}_{\alpha} X_{j: n}-\mu}{\sigma_{1}}=\frac{K_{j: n}(\alpha)}{M_{1}(\alpha)}, \quad 0 \leq \alpha<1 .
$$

Moreover,

$$
K_{j: n}^{\prime}(\alpha)=\alpha\left[1-f_{j: n}(\alpha)\right], \quad 0 \leq \alpha<1
$$

This means that for $2 \leq j \leq n-1$ function $K_{j: n}(\alpha)$ increases from $\frac{j}{n+1}-\frac{1}{2}$ at 0 to $K_{j: n}\left(a_{j: n}\right)$, then decreases to $K_{j: n}\left(b_{j: n}\right)$, and ultimately increases to 0 at 1 . Function (7) is strictly positive for $0 \leq \alpha<1$, and tends to 0 as $\alpha \nearrow 1$. By the de l'Hospital rule, we also have 


$$
\lim _{\alpha \nearrow 1} \frac{K_{j: n}(\alpha)}{M_{1}(\alpha)}=\lim _{\alpha \nearrow 1} \frac{\alpha\left[1-f_{j: n}(\alpha)\right]}{1-3 \alpha^{2}}=-\frac{1}{2}
$$

Therefore $K_{j: n}\left(a_{j: n}\right)=0$ implies

$$
\sup _{0 \leq \alpha<1} \frac{K_{j: n}(\alpha)}{M_{1}(\alpha)}=\frac{K_{j: n}\left(a_{j: n}\right)}{M_{1}\left(a_{j: n}\right)}=0 \geq \sup _{F \in D D A(1)} \frac{\mathbb{E}_{F} X_{j: n}-\mu}{\sigma_{1}},
$$

which verifies (10). One should note, though, that equality $K_{j: n}\left(a_{j: n}\right)=0$ for some $(j, n)$ is very unlikely, because $a_{j: n}$ is usually an irrational number, and $K_{j: n}$ is a polynomial with rational coefficients.

Now we prove that

$$
\frac{\mathbb{E}_{F} X_{j: n}-\mu}{\sigma_{1}}<0, \quad F \in D D A(1),
$$

implies

$$
\sup _{F \in D D A(1)} \frac{\mathbb{E}_{F} X_{j: n}-\mu}{\sigma_{1}}=\sup _{0 \leq \alpha<1} \frac{\mathbb{E}_{\alpha} X_{j: n}-\mu}{\sigma_{1}}=\sup _{0 \leq \alpha<1} \frac{K_{j: n}(\alpha)}{M_{1}(\alpha)}<0 .
$$

We can write

$$
\frac{\mathbb{E}_{F} X_{j: n}-\mu}{\sigma_{1}}=\int_{0}^{1} \frac{F^{-1}(x)-\mu}{\sigma_{1}}\left[f_{j: n}(x)-1\right] d x=\int_{0}^{1} g(x) f(x) d x=T_{f}(g) .
$$

Function $f=f_{j: n}-1$ is fixed and integrates to 0 . Functions $g=\frac{F^{-1}-\mu}{\sigma_{1}}$, $F \in D D A(1)$, belong to the subset $\mathcal{G}$ of elements of $L^{1}([0,1), d x)$ which are (right-continuous versions of) non-decreasing, starshaped functions with unit norms satisfying

$$
T_{1}(g)=\int_{0}^{1} g(x) d x=\int_{0}^{1} \frac{F^{-1}(x)-\mu}{\sigma_{1}} d x=0 .
$$

In particular,

$$
g_{\alpha}(x)=\frac{1}{M_{1}(\alpha)}\left[x \mathbf{1}_{[\alpha, 1)}(x)-\frac{1-\alpha^{2}}{2}\right] \in \mathcal{G}, \quad 0 \leq \alpha<1 .
$$

Since $T_{f}(g)<0$ for $f \in D D A(1)$, the simple linear operator $g \mapsto \tilde{g}=\frac{g}{-T_{f}(g)}$ transforms $\mathcal{G}$ onto the convex subset $\tilde{\mathcal{G}}$ of $L^{1}([0,1), d x)$ whose elements are rightcontinuous, non-decreasing, star-shaped, and satisfy 


$$
\begin{aligned}
& T_{1}(\tilde{g})=\int_{0}^{1} \tilde{g}(x) d x=0, \\
& T_{f}(\tilde{g})=\int_{0}^{1} \tilde{g}(x) f(x) d x=-1 .
\end{aligned}
$$

In particular,

$$
\tilde{g}_{\alpha}(x)=\frac{-1}{K_{j: n}(\alpha)}\left[x \mathbf{1}_{[\alpha, 1)}(x)-\frac{1-\alpha^{2}}{2}\right] \in \tilde{\mathcal{G}}, \quad 0 \leq \alpha<1 .
$$

Note that $\|\tilde{g}\|=\frac{1}{-T_{f}(g)}, \tilde{g} \in \tilde{\mathcal{G}}$, and the problem of maximizing $T_{f}(g), g \in \mathcal{G}$, is equivalent with that of maximizing $\|\tilde{g}\|, \tilde{g} \in \tilde{\mathcal{G}}$. The norm functional is convex, and so

$$
\left\|\alpha \tilde{g}_{1}+(1-\alpha) \tilde{g}_{2}\right\| \leq \alpha\left\|\tilde{g}_{1}\right\|+(1-\alpha)\left\|\tilde{g}_{2}\right\| \leq \max \left\{\left\|\tilde{g}_{1}\right\|,\left\|\tilde{g}_{2}\right\|\right\}
$$

for arbitrary $\tilde{g}_{1}, \tilde{g}_{2} \in \tilde{\mathcal{G}}$ and $0 \leq \alpha \leq 1$.

Suppose that for some $0<\alpha_{1}<\cdots<\alpha_{k}<\alpha_{k+1}=1$, and $\beta_{-1}<\beta_{0}=0<$ $\beta_{1}<\cdots<\beta_{k}$

$$
h_{\alpha, \beta}(x)=\beta_{-1}+\sum_{i=1}^{k} \beta_{i} x \mathbf{1}_{\left[\alpha_{i}, \alpha_{i+1}\right)}(x) \in \tilde{\mathcal{G}}
$$

We show that $h_{\alpha, \beta}$ is a convex combination of functions $\tilde{g}_{\alpha_{i}}, i=1, \ldots, k$. Firstly, $\tilde{g}_{\alpha_{i}}, i=1, \ldots, k$, are linearly independent. Their linear combinations $\tilde{h}_{\alpha, \gamma}(x)=$ $\sum_{i=1}^{k} \gamma_{i} \tilde{g}_{\alpha_{i}}(x)$ satisfy

$$
T_{1}\left(\tilde{h}_{\alpha, \gamma}\right)=\int_{0}^{1} \tilde{h}_{\alpha, \gamma}(x) d x=0
$$

and

$$
\frac{\tilde{h}_{\alpha, \gamma}(x)-\tilde{h}_{\alpha, \gamma}(0)}{x}=\sum_{i=1}^{k} \frac{\gamma_{i}}{K_{j: n}\left(\alpha_{i}\right)} \mathbf{1}_{\left[\alpha_{i}, 1\right)}(x)=\sum_{i=1}^{k} \delta_{i} \mathbf{1}_{\left[\alpha_{i-1}, \alpha_{i}\right)}(x)
$$

for $\delta_{j}=\sum_{i=1}^{j} \frac{\gamma_{i}}{K_{j: n}\left(\alpha_{i}\right)}, j=1, \ldots, k$. In particular,

$$
h_{\alpha, \beta}(x)=\sum_{i=1}^{k} K_{i: n}\left(\alpha_{i}\right)\left(\beta_{i}-\beta_{i-1}\right) \tilde{g}_{\alpha_{i}}(x)=\sum_{i=1}^{k} \gamma_{i} \tilde{g}_{\alpha_{i}}(x) .
$$


Starshapedness of $h_{\alpha, \beta}$ and condition

$$
-1=T_{f}\left(h_{\alpha, \beta}\right)=\sum_{i=1}^{k} \gamma_{i} T_{f}\left(\tilde{g}_{\alpha_{i}}\right)=-\sum_{i=1}^{k} \gamma_{i}
$$

imply that $\gamma_{i}, i=1, \ldots, k$, are non-negative, and sum up to 1 . In consequence,

$$
\left\|h_{\alpha, \beta}\right\| \leq \max _{1 \leq i \leq k}\left\|\tilde{g}_{\alpha_{i}}\right\|
$$

Consider now arbitrary $\tilde{g} \in \tilde{\mathcal{G}}$. For $k=1,2 \ldots$, define

$$
g_{k}(x)=g(0)+\sum_{i=1}^{2^{k}-1} \frac{2^{k}}{i}\left[g\left(i / 2^{k}\right)-g(0)\right] x \mathbf{1}_{\left[i / 2^{k},(i+1) / 2^{k}\right)}(x) .
$$

The functions are non-decreasing, starshaped, and tend monotonously to $\tilde{g}$ on $[0,1)$. By the Lebesgue monotone convergence theorem,

$$
\left\|\tilde{g}-g_{k}\right\|=\int_{0}^{1}\left[\tilde{g}(x)-g_{k}(x)\right] d x \searrow 0
$$

and in consequence $T_{1}\left(g_{k}\right) \rightarrow 0$, and $T_{f}\left(g_{k}\right) \rightarrow-1$ as $k \rightarrow \infty$. Furthermore, each

$$
\tilde{g}_{k}=\frac{g_{k}-T_{1}\left(g_{k}\right)}{-T_{f}\left(g_{k}\right)} \in \tilde{\mathcal{G}},
$$

and so is a convex combination of $\tilde{g}_{i / 2^{k}}, i=1, \ldots, 2^{k}-1$, and

$$
\begin{aligned}
\left\|\tilde{g}_{k}-\tilde{g}\right\| & =|| \frac{g_{k}}{-T_{f}\left(g_{k}\right)}-g_{k}+g_{k}-\tilde{g}-\frac{T_{1}\left(g_{k}\right)}{-T_{f}\left(g_{k}\right)} \| \\
& \leq\left|\frac{1}{-T_{f}\left(g_{k}\right)}-1\right|\left\|g_{k}\right\|+\left\|g_{k}-\tilde{g}\right\|+\frac{\left|T_{1}\left(g_{k}\right)\right|}{-T_{f}\left(g_{k}\right)} \rightarrow 0 .
\end{aligned}
$$

By continuity of the norm functional,

$$
\begin{aligned}
& \max _{i=1, \ldots, 2^{k}-1}\left\|\tilde{g}_{i / 2^{k}}\right\| \geq\left\|\tilde{g}_{k}\right\| \rightarrow\|\tilde{g}\|, \\
& \sup _{0 \leq \alpha<1}\left\|\tilde{g}_{\alpha}\right\| \geq\|\tilde{g}\|, \quad \tilde{g} \in \tilde{\mathcal{G}},
\end{aligned}
$$

and in consequence

$$
\sup _{0 \leq \alpha<1} \frac{K_{j: n}(\alpha)}{M_{1}(\alpha)}=\sup _{0 \leq \alpha<1} T_{f}\left(g_{\alpha}\right) \geq \sup _{g \in \mathcal{G}} T_{f}(g)=\sup _{F \in D D A(1)} \frac{\mathbb{E}_{F} X_{j: n}-\mu}{\sigma_{1}}
$$

So we proved that (13) implies (14). 
If $j=1$ and $\sigma_{1}>0$, we have (13). By (12), $K_{1: n}(\alpha)<0,0 \leq \alpha<1$, and so $\sup _{0 \leq \alpha<1} \frac{K_{1: n}(\alpha)}{M_{1}(\alpha)}<0$. If $j \geq 2$ and $K_{j: n}\left(a_{j: n}\right)=0$, then condition (13) does not hold. Then

$$
\sup _{F \in D D A(1)} \frac{\mathbb{E}_{F} X_{j: n}-\mu}{\sigma_{1}}=\frac{\mathbb{E}_{a_{j: n}} X_{j: n}-\mu}{\sigma_{1}}=0
$$

Summing up, under condition $K_{j: n}\left(a_{j: n}\right) \leq 0$, we have

$$
\sup _{F \in D D A(1)} \frac{\mathbb{E}_{F} X_{j: n}-\mu}{\sigma_{1}}=\sup _{0 \leq \alpha<1} \frac{K_{j: n}(\alpha)}{M_{1}(\alpha)} \leq 0
$$

and the attainability conditions coincide with ones presented in Proposition 3.

By Proposition 3,

$$
\sup _{F \in D D A(1)} \frac{\mathbb{E}_{F} X_{j: n}-\mu}{\sigma_{1}} \geq \lim _{\alpha \nearrow 1} \frac{K_{j: n}(\alpha)}{M_{1}(\alpha)}=-\frac{1}{2} .
$$

It occurs that the supremum is equal to $-\frac{1}{2}$ for some small $j$ close to 1 . These bounds are attained in the limit. For larger $j$, the negative bounds fall between $-\frac{1}{2}$ and 0 , and they are attained by particular combinations of the degenerate and uniform distributions. These combinations always contain atoms, because for the uniform distribution we have

$$
\frac{\mathbb{E}_{0} X_{j: n}-\mu}{\sigma_{1}}=\frac{K_{j: n}(0)}{M_{1}(0)}=4\left(\frac{j}{n+1}-\frac{1}{2}\right) \geq 4\left(\frac{1}{n+1}-\frac{1}{2}\right)>-\frac{1}{2}
$$

when $n \geq 2$. Observe that condition $\frac{K_{j: n}(0)}{M_{1}(0)}=4\left(\frac{j}{n+1}-\frac{1}{2}\right) \leq 0$ is necessary for nonpositivity of the bounds in the DD case. This is the necessary and sufficient for nonpositivity of $\mathbb{E}_{F} X_{j: n}-\mu$ for the parent distributions with decreasing density functions (cf Rychlik 2009b). Numerical examples show that conditions $K_{j: n}\left(a_{j: n}\right) \leq 0$ and $j \leq \frac{n+1}{2}$ do not differ much for small $n$.

Table 1 contains exemplary values of negative, greater than $-\frac{1}{2}$, tight upper bounds on the expectations of standardized order statistics $\mathbb{E}_{F}\left(X_{j: n}-\mu\right) / \sigma_{1}$ for $n=10,20,30$. If for a given $n$, the numerical bounds are presented for $j=$ $j_{1}(n), \ldots, j_{2}(n)$, one should conclude that the respective bounds are equal to $-\frac{1}{2}$ for $j<j_{1}(n)$, and are positive for $j>j_{2}(n)$. Each bound is accompanied by the value of argument $\alpha$ which maximizes the RHS of (9) for a given pair $(j, n)$. It fully determines the mixture distribution for which the bound is attained. The simplest interpretation of this parameter is that it is equal to the probability of the atom in the optimal mixture. In the cases of our numerical analysis the atom probabilities range between 0.1817 and 0.2988 . 
Table 1 Negative upper bounds on the expectations of standardized order statistics $\left(\mathbb{E} X_{j: n}-\mu\right) / \sigma_{1}$, $n=10,20,30$, coming from DDA populations

\begin{tabular}{llllllll}
\hline$n$ & $j$ & Bound & Atom prob. & $n$ & $j$ & Bound & Atom prob. \\
\hline 10 & 4 & -0.496507 & 0.181698 & \multirow{2}{*}{30} & 11 & -0.474085 & 0.222497 \\
& 5 & -0.105490 & 0.244825 & & 12 & -0.328868 & 0.248340 \\
20 & 8 & -0.381335 & 0.225906 & & 13 & -0.186415 & 0.273790 \\
& 9 & -0.172557 & 0.261855 & & 14 & -0.047683 & 0.298779 \\
\hline
\end{tabular}

\section{DFRA distributions}

The assumptions in Sect. 3 are following.

Assumptions $D F R A(p)$ : Let $X_{1}, \ldots, X_{n}$ be i.i.d. random variables with a parent distribution function $F$ which has the DFRA property, i.e. its support starts at a finite point $a_{F}$, and the ratio $-\ln (1-F(x)) /\left(x-a_{F}\right)$ is nonincreasing for $x>a_{F}$. Each $X_{i}$ has a mean $\mu \in \mathbb{R}$, and $p$ th central absolute moment $0<\sigma_{p}^{p}<\infty$ for some $1 \leq p<\infty$. Further we assume that either $j=1$ or $2 \leq j \leq n-1$ which satisfies $L_{j: n}\left(a_{j: n}\right) \leq 0$, where $L_{j: n}$ is defined in (5), and $a_{j: n}$ is the smaller of two roots of $f_{j: n}(x)=1$ belonging to $(0,1)$. We define $N_{p}(\alpha)=\left(N_{p}^{p}(\alpha)\right)^{1 / p}, p \geq 1, \alpha \geq 0$, where

$$
\begin{aligned}
N_{p}^{p}(\alpha)= & (\alpha+1)^{p} e^{-p \alpha}\left(1-e^{-\alpha}\right)+\exp \left(-(\alpha+1) e^{-\alpha}\right) \\
& \times \begin{cases}{\left[\int_{0}^{(\alpha+1) e^{-\alpha}-\alpha} x^{p} e^{x} d x+\Gamma(p+1)\right],} & \alpha \leq \alpha_{*}, \\
\int_{\alpha-(\alpha+1) e^{-\alpha}}^{\infty} x^{p} e^{-x} d x, & \alpha \geq \alpha_{*},\end{cases}
\end{aligned}
$$

with $\alpha_{*} \approx 0.80647$ uniquely determined by the equation $\alpha e^{\alpha}=\alpha+1$. We also use here

$$
Q_{1}(\beta)=N_{1}(-\ln (1-\beta))= \begin{cases}2\left(\frac{1-\beta}{e}\right)^{1-\beta}, & 0 \leq \beta \leq \beta_{*}, \\ 2 \beta(1-\beta)[1-\ln (1-\beta)], & \beta_{*} \leq \beta<1,\end{cases}
$$

with $\beta_{*}=1-e^{-\alpha_{*}} \approx 0.408156$ being the solution to the equation $-\ln (1-\beta)=\frac{1}{\beta}-1$.

Proposition 4 (Case $p>1$ ) If assumptions DFRA(p) are satisfied for some $p>1$ and $1 \leq j<n$, then the evaluation

$$
\frac{\mathbb{E}_{F} X_{j: n}-\mu}{\sigma_{p}} \leq 0
$$

is sharp, and the equality is attained in the limit by the family of mixtures of the atom at $\mu-\frac{\sigma_{p}(1-\beta)[1-\ln (1-\beta)]}{N_{p}(-\ln (1-\beta))}$ with probability $\beta$, and the exponential distribution with location $\mu+\frac{\sigma_{p}[-(1-\beta)-\ln (1-\beta)]}{N_{p}(-\ln (1-\beta))}$ and scale $\frac{\sigma_{p}}{N_{p}(-\ln (1-\beta))}$ with probability $1-\beta$, as $\beta \nearrow 1$. 
Proof It only suffices to check the attainability conditions. For the family of mixture distributions, the the quantile functions satisfy

$$
\frac{F_{\beta}^{-1}(y)-\mu}{\sigma_{p}}=\frac{-\ln (1-y) \mathbf{1}_{[\beta, 1)}(y)-(1-\beta)[1-\ln (1-\beta)]}{N_{p}(-\ln (1-\beta))} .
$$

Substituting $x=-\ln (1-y)$ and setting $\alpha=-\ln (1-\beta)$, we obtain

$$
\frac{F_{\beta}^{-1}\left(1-e^{-x}\right)-\mu}{\sigma_{p}}=\frac{x \mathbf{1}_{[\alpha, \infty)}(x)-(\alpha+1) e^{-\alpha}}{N_{p}(\alpha)} .
$$

This shows that $F_{\beta}, 0 \leq \beta<1$, are DFRA. Since

$$
\begin{aligned}
\mathbb{E}_{\beta} X_{1}-\mu & =\int_{0}^{1}\left[F_{\beta}^{-1}(y)-\mu\right] d y=\int_{0}^{\infty}\left[F_{\beta}^{-1}\left(1-e^{-x}\right)-\mu\right] e^{-x} d x \\
& =\frac{\sigma_{p}}{N_{p}(\alpha)} \int_{0}^{\infty}\left[x \mathbf{1}_{[\alpha, \infty)}(x)-(\alpha+1) e^{-\alpha}\right] e^{-x} d x=0,
\end{aligned}
$$

and

$$
\begin{aligned}
& \frac{N_{p}^{p}(\alpha)}{\sigma_{p}^{p}} \mathbb{E}_{\beta}\left|X_{1}-\mu\right|^{p}=\frac{N_{p}^{p}(\alpha)}{\sigma_{p}^{p}} \int_{0}^{1}\left|F_{\beta}^{-1}(y)-\mu\right|^{p} d y \\
& =\int_{0}^{\infty}\left|x \mathbf{1}_{[\alpha, \infty)}(x)-(\alpha+1) e^{-\alpha}\right|^{p} e^{-x} d x \\
& =\int_{0}^{\alpha}(\alpha+1)^{p} e^{-p \alpha} e^{-x} d x+\exp \left(-(\alpha+1) e^{-\alpha}\right) \int_{\alpha-(\alpha+1) e^{-\alpha}}^{\infty}|x|^{p} e^{-x} d x \\
& =(\alpha+1)^{p} e^{-p \alpha}\left(1-e^{-\alpha}\right)+\exp \left(-(\alpha+1) e^{-\alpha}\right) \quad \alpha \geq \alpha_{*}, \quad \alpha_{*}, \\
& \quad \times\left\{\begin{array}{l}
\left.\int_{\alpha-(\alpha+1) e^{-\alpha}}^{0}(-x)^{p} e^{-x} d x+\int_{0}^{\infty} x^{p} e^{-x} d x, \quad \alpha\right), \\
\int_{\alpha-(\alpha+1) e^{-\alpha}}^{\infty} x^{p} e^{-x} d x,
\end{array}\right.
\end{aligned}
$$

distribution functions $F_{\beta}, 0 \leq \beta<1$, satisfy both the moment conditions. Furthermore, we calculate

$$
\begin{aligned}
& \frac{N_{p}(\alpha)}{\sigma_{p}}\left(\mathbb{E}_{\beta} X_{j: n}-\mu\right)=\frac{N_{p}(\alpha)}{\sigma_{p}} \int_{0}^{1}\left[F_{\beta}^{-1}(y)-\mu\right] f_{j: n}(y) d y \\
& \quad=\int_{0}^{\infty}\left[x \mathbf{1}_{[\alpha, \infty)}(x)-(\alpha+1) e^{-\alpha}\right] f_{j: n}\left(1-e^{-x}\right) e^{-x} d x
\end{aligned}
$$




$$
\begin{aligned}
& \int_{\alpha}^{\infty} x f_{j: n}\left(1-e^{-x}\right) e^{-x} d x-(\alpha+1) e^{-\alpha} \\
= & \alpha \bar{F}_{j: n}\left(1-e^{-\alpha}\right)+\int_{\alpha}^{\infty} \bar{F}_{j: n}\left(1-e^{-x}\right) d x-(\alpha+1) e^{-\alpha} \\
= & \sum_{i=0}^{j-1}\left(\begin{array}{c}
n \\
i
\end{array}\right) \int_{\alpha}^{\infty}\left(1-e^{-x}\right)^{i} e^{-(n-1-i) x} e^{-x} d x+\alpha \bar{F}_{j: n}\left(1-e^{-\alpha}\right)-(\alpha+1) e^{-\alpha} \\
= & \sum_{i=0}^{j-1} \frac{1}{n-i} \int_{1-e^{-\alpha}}^{1} f_{i+1: n}(y) d y+\alpha \bar{F}_{j: n}\left(1-e^{-\alpha}\right)-(\alpha+1) e^{-\alpha} \\
= & \sum_{i=1}^{j} \frac{\bar{F}_{i: n}\left(1-e^{-x}\right)}{n+1-i}+\alpha \bar{F}_{j: n}\left(1-e^{-\alpha}\right)-(\alpha+1) e^{-\alpha} \\
= & L_{j: n}\left(1-e^{-\alpha}\right) \leq 0 .
\end{aligned}
$$

Accordingly, it remains to prove that for $p>1$

$$
\lim _{\beta \nearrow 1} \frac{\mathbb{E}_{\beta} X_{j: n}-\mu}{\sigma_{p}}=\lim _{\alpha \nearrow \infty} \frac{L_{j: n}\left(1-e^{-\alpha}\right)}{N_{p}(\alpha)}=0 .
$$

We observe that

$$
\lim _{\alpha \nearrow \infty} \frac{L_{j: n}\left(1-e^{-\alpha}\right)}{\alpha e^{-\alpha}}=-1,
$$

because

$$
\begin{aligned}
\lim _{\alpha \nearrow \infty} \sum_{i=1}^{j} \frac{\bar{F}_{i: n}\left(1-e^{-x}\right)}{(n+1-i) e^{-(n+1-j) \alpha}} & =\left(\begin{array}{c}
n \\
j-1
\end{array}\right) \frac{1}{n+1-j}, \\
\lim _{\alpha \nearrow \infty} \frac{\alpha \bar{F}_{j: n}\left(1-e^{-\alpha}\right)}{\alpha e^{-(n+1-j) \alpha}} & =\left(\begin{array}{c}
n \\
j-1
\end{array}\right), \\
\lim _{\alpha \nearrow \infty} \frac{(\alpha+1) e^{-\alpha}}{\alpha e^{-\alpha}} & =1 .
\end{aligned}
$$

Also,

$$
\lim _{\alpha \nearrow \infty} \frac{N_{p}^{p}(\alpha)}{\alpha^{p} e^{-\alpha}}=1
$$


because

$$
\begin{aligned}
\lim _{\alpha \nearrow \infty} \frac{(\alpha+1)^{p} e^{-p \alpha}\left(1-e^{-\alpha}\right)}{\alpha^{p} e^{-p \alpha}} & =1, \\
\lim _{a \nearrow \infty} \frac{\int_{a}^{\infty} x^{p} e^{-x} d x}{a^{p} e^{-a}} & =\lim _{a \nearrow \infty} \frac{-a^{p} e^{-a}}{p a^{p-1} e^{-a}-a^{p} e^{-a}} \\
& =\lim _{a \nearrow \infty} \frac{1}{1-p / a}=1, \\
\lim _{\alpha \nearrow \infty} \frac{\exp \left(-(\alpha+1) e^{-\alpha}\right) \int_{\alpha-(\alpha+1) e^{-\alpha}}^{\infty} x^{p} e^{-x} d x}{\alpha^{p} e^{-\alpha}} & =\lim _{\alpha \nearrow \infty} \frac{\left(\alpha-(\alpha+1) e^{-\alpha}\right)^{p} e^{-\alpha}}{\alpha^{p} e^{-\alpha}}=1 .
\end{aligned}
$$

Therefore

$$
\lim _{\alpha \nearrow \infty} \frac{L_{j: n}\left(1-e^{-\alpha}\right)}{N_{p}(\alpha)}=\lim _{\alpha \nearrow \infty} \frac{\alpha e^{-\alpha}}{\alpha e^{-\alpha / p}}=0,
$$

which ends the proof.

Proposition 5 (Case $p=1$ ) Under the assumptions DFRA $(p)$ with $p=1$, we have

$$
\frac{\mathbb{E}_{F} X_{j: n}-\mu}{\sigma_{p}} \leq \sup _{0 \leq \beta<1} \frac{L_{j: n}(\beta)}{Q_{1}(\beta)},
$$

where the numerator and denominator of the RHS are defined in (5) and (15), respectively. If the supremum of the RHS of (16) is attained at some $0 \leq \beta<1$, then the bound is attained by the mixture of the pole at $\mu-\frac{\sigma_{1}(1-\beta)[1-\ln (1-\bar{\beta})]}{\left.Q_{1}(\beta)\right)}$ with probability $\beta$, and the exponential distribution with location $\mu+\frac{\sigma_{1}[-(1-\beta)-\ln (1-\beta)]}{Q_{1}(\beta)}$ and scale $\frac{\sigma_{1}}{Q_{1}(\beta)}$ with probability $1-\beta$. Otherwise, i.e. when

$$
\sup _{0 \leq \beta<1} \frac{L_{j: n}(\beta)}{Q_{1}(\beta)}=\lim _{\beta \nearrow 1} \frac{L_{j: n}(\beta)}{Q_{1}(\beta)}=-\frac{1}{2},
$$

then the bound is attained in the limit by the sequences of the mixture distributions as $\beta \nearrow 1$.

Proof It is similar to that of Proposition 3, and we describe only the main ideas. Recalling the arguments of the proof of Proposition 4, we observe that the mixture distributions described in Proposition 5 are DFRA, and have first raw and central absolute moments $\mu$ and $\sigma_{1}$, respectively, for all $0 \leq \beta<1$. Moreover,

$$
\frac{\mathbb{E}_{\beta} X_{j: n}-\mu}{\sigma_{1}}=\frac{L_{j: n}(\beta)}{Q_{1}(\beta)}, \quad 0 \leq \beta<1,
$$

holds. Since

$$
L_{j: n}^{\prime}(\beta)=-\ln (1-\beta)\left[1-f_{j: n}(\beta)\right]
$$


function $L_{j: n}$ itself is first decreasing, and then increasing for $j=1$, and it is increasing on $\left[0, a_{j: n}\right]$, decreasing on $\left[a_{j: n}, b_{j: n}\right]$, and ultimately increasing for $j \geq 2$. Function (15) is strictly positive. Since

$$
\lim _{\beta \nearrow 1} \frac{L_{j: n}(\beta)}{Q_{1}(\beta)}=\lim _{\beta \nearrow 1} \frac{-\ln (1-\beta)\left[1-f_{j: n}(\beta)\right]}{2(1-2 \beta)[-\ln (1-\beta)]+2 \beta}=-\frac{1}{2},
$$

the supremum of the RHS in (16) is non-negative iff $L_{j: n}\left(a_{j: n}\right) \geq 0$, and this certainly implies that

$$
\sup _{F \in D F R A(1)} \frac{\mathbb{E}_{F} X_{j: n}-\mu}{\sigma_{1}} \geq 0
$$

Now we show that under the condition

$$
\frac{\mathbb{E}_{F} X_{j: n}-\mu}{\sigma_{1}}<0, \quad F \in \operatorname{DFRA}(1)
$$

(that implies $L_{j: n}\left(a_{j: n}\right)<0$ in particular), we have

$$
\sup _{F \in D F R A(1)} \frac{\mathbb{E}_{F} X_{j: n}-\mu}{\sigma_{1}}=\sup _{0 \leq \beta<1} \frac{\mathbb{E}_{\beta} X_{j: n}-\mu}{\sigma_{1}}=\sup _{0 \leq \beta<1} \frac{L_{j: n}(\beta)}{Q_{1}(\beta)}<0 .
$$

First we note that

$$
\begin{aligned}
\frac{\mathbb{E}_{F} X_{j: n}-\mu}{\sigma_{1}} & =\int_{0}^{\infty} \frac{F^{-1}\left(1-e^{-x}\right)-\mu}{\sigma_{1}}\left[f_{j: n}\left(1-e^{-x}\right)-1\right] e^{-x} d x \\
& =\int_{0}^{\infty} h(x) f(x) e^{-x} d x,
\end{aligned}
$$

where $f(x)=f_{j: n}\left(1-e^{-x}\right)-1$, and $h(x)=\frac{F^{-1}\left(1-e^{-x}\right)-\mu}{\sigma_{1}}$ is an arbitrary element of the set $\mathcal{H} \subset L^{2}\left(\mathbb{R}_{+}, e^{-x} d x\right)$, which contains the (right-continuous versions of) non-decreasing, starshaped functions such that

$$
\begin{aligned}
S_{1}(h) & =\int_{0}^{\infty} h(x) e^{-x} d x=0, \\
\|h\|= & \int_{0}^{\infty}|h(x)| e^{-x} d x=1 .
\end{aligned}
$$


We also have

$$
S_{f}(h)=\int_{0}^{\infty} h(x) f(x) e^{-x} d x<0, \quad h \in \mathcal{H} .
$$

The family $\tilde{\mathcal{H}}$ of functions $\tilde{h}(x)=\frac{h(x)}{-S_{f}(h)}$ is a convex set in $L^{2}\left(\mathbb{R}_{+}, e^{-x} d x\right)$ which consists of non-decreasing starshaped functions with $S_{1}(\tilde{h})=0$ and $S_{f}(\tilde{h})=-1$. Since $\|\tilde{h}\|=-\frac{1}{S_{f}(h)}$, the problems of maximizing $S_{f}$ over $\mathcal{H}$ and that of maximizing the norm over $\tilde{\mathcal{H}}$ are equivalent.

Using the convexity property of the norm functional, we prove that the latter problem is solved by the elements

$$
\tilde{h}_{\alpha}(x)=\frac{x \mathbf{1}_{[\alpha, \infty)}(x)-(\alpha+1) e^{-\alpha}}{L_{j: n}(\alpha)}, \quad 0 \leq \alpha<\infty,
$$

of a parametric class contained in $\tilde{\mathcal{H}}$. Every $\tilde{h} \in \tilde{\mathcal{H}}$ can be approximated by piecewise linear discontinuous functions

$$
\begin{aligned}
h_{k}(x)= & \tilde{h}(0)+\sum_{i=1}^{4^{k}-1} \frac{2^{k}}{i}\left[\tilde{h}\left(\frac{i}{2^{k}}\right)-\tilde{h}(0)\right] x \mathbf{1}_{\left[\frac{i}{2^{k}}, \frac{i+1}{2^{k}}\right)}(x) \\
& +\frac{\tilde{h}\left(2^{k}\right)-\tilde{h}(0)}{2^{k}} \times \mathbf{1}_{\left[2^{k}, \infty\right)}(x)
\end{aligned}
$$

$k=1,2, \ldots$, so that $h_{k} \nearrow \tilde{h}$ and $\left\|\tilde{h}-h_{k}\right\| \searrow 0$ as $k \rightarrow \infty$. Functions

$$
\tilde{h}_{k}=\frac{h_{k}-S_{1}\left(h_{k}\right)}{-S_{f}\left(h_{k}\right)} \in \tilde{\mathcal{H}},
$$

and are convex combinations of $\tilde{h}_{\frac{i}{2^{k}}}, i=1, \ldots, 4^{k}$. We have

$$
\|\tilde{h}\| \leftarrow\left\|\tilde{h}_{k}\right\| \leq \sup _{1 \leq i \leq 4^{k}}\left\|\tilde{h}_{\frac{i}{2^{k}}}\right\| \leq \sup _{0 \leq \alpha<\infty}\left\|\tilde{h}_{\alpha}\right\| .
$$

This allows us to deduce (17) which ends the proof.

As in the DDA case, under condition (5) yields

$$
-\frac{1}{2} \leq \sup _{F \in D F R A(1)} \frac{\mathbb{E}_{F} X_{j: n}-\mu}{\sigma_{1}} \leq 0 .
$$

The supremum is not attained by the exponential distribution (without any contribution of the atom) because 
Table 2 Negative upper bounds on the expectations of standardized order statistics $\left(\mathbb{E} X_{j: n}-\mu\right) / \sigma_{1}$, $n=10,20,30$, coming from DFRA populations

\begin{tabular}{rrllllll}
\hline$n$ & $j$ & Bound & Atom prob. & $n$ & $j$ & Bound & Atom prob. \\
\hline 10 & 7 & -0.394763 & 0.713069 & 30 & 18 & -0.481136 & 0.570305 \\
& 8 & -0.175588 & 0.786236 & & 19 & -0.399720 & 0.596811 \\
20 & 13 & -0.415996 & 0.627060 & & 20 & -0.316247 & 0.625045 \\
& 14 & -0.299618 & 0.666699 & & 21 & -0.228813 & 0.654837 \\
& 15 & -0.170139 & 0.710833 & & 22 & -0.134745 & 0.686115 \\
& 16 & -0.015518 & 0.759193 & & 23 & -0.030140 & 0.718871 \\
\hline
\end{tabular}

$$
\frac{L_{j: n}(0)}{Q_{1}(0)}=\frac{e}{2}\left(\sum_{i=1}^{j} \frac{1}{n+1-i}-1\right)>\frac{e}{2}\left(\frac{1}{n}-1\right)>-\frac{1}{2}
$$

for all $n \geq 2$. The sign of $\frac{L_{j: n}(0)}{Q_{1}(0)}$ is identical with the sign of the upper bound on $\frac{\mathbb{E}_{F} X_{j: n}-\mu}{\sigma_{1}}$ for the DFR populations (cf Rychlik 2002, 2009c).

Table 2 provides the tight upper negative and different from $-\frac{1}{2}$ bounds on the standardized expectations of order statistics from DFRA populations, when the sample size $n=10,20$, and 30. If for a given $n$, the bounds are presented for $j=j_{1}(n), \ldots, j_{2}(n)$, then the respective evaluations amount to $-\frac{1}{2}$ for $j=1, \ldots, j_{1}(n)-1$, and are positive for $j_{2}(n)+1, \ldots, n$. Together with the bounds values, there are listed the probabilities of atoms in the DFRA distributions attaining the bounds. They are essentially greater than the respective values in Table 1 for the DDA distributions, and greater than the contributions of the uniform distributions in the mixtures as well.

We finally note that we presented simple examples of families of distributions which attain in the limit the zero bounds in Propositions 2 and 4, and $-\frac{1}{2}$ in Propositions 3 and 5. Slight modifications of the distributions would not violate these asymptotic properties. The other bounds determined in Propositions 3 and 5 are attained by the distributions uniquely determined up to the location and scale transformations.

Acknowledgments The research was supported by the Polish National Science Center Grant no. N N201 416739 .

Open Access This article is distributed under the terms of the Creative Commons Attribution License which permits any use, distribution, and reproduction in any medium, provided the original author(s) and the source are credited.

\section{References}

Arnold BC (1985) p-Norm bounds on the expectation of the maximum of possibly dependent sample. J Multivar Anal 17:316-332

Arnold BC, Balakrishnan N (1989) Relations, bounds and approximations for order statistics. Lecture notes in statistics, vol 53. Springer, New York

Barlow RE, Proschan F (1966) Inequalities for linear combinations of order statistics from restricted families. Ann Math Stat 37:1574-1592 
Barlow RE, Marshall AW, Proschan F (1969) Some inequalities for starshaped and convex functions. Pac J Math 29:19-42

Bieniek M (2006) Projection bounds on expectations of generalized order statistics from DFR and DFRA families. Statistics 40:339-352

Bieniek M (2008) Projection bounds on expectations of generalized order statistics from DD and DDA families. J Stat Plan Inference 138:971-981

Danielak K (2003) Sharp upper bounds on trimmed means from restricted families. Statistics 37:305-324

Danielak K, Rychlik T (2003) Sharp bounds for expectations of spacings from DDA and DFRA families. Stat Probab Lett 65:303-316

Danielak K, Rychlik T (2004) Sharp bounds for expectations of spacings from decreasing density and failure rate families. Appl Math (Warsaw) 31:369-395

Gajek L, Rychlik T (1998) Projection method for moment bounds on order statistics from restricted families. II. Independent case. J Multivar Anal 64:156-182

Goroncy A (2009) Lower bounds on positive $L$-statistics. Commun Stat Theory Meth 38:1989-2002

Goroncy A (2013) Bounds on expected generalized order statistics. Statistics. doi.10.1080/02331888.2013. 800073

Gumbel EJ (1954) The maxima of the mean largest value and of the range. Ann Math Stat 25:76-84

Hartley HO, David HA (1954) Universal bounds for mean range and extreme observation. Ann Math Stat 25:85-99

Moriguti S (1953) A modification of Schwarz's inequality with applications to distributions. Ann Math Stat 24:107-113

Rychlik T (1998) Bounds for expectations of $L$-estimates. In: Balakrishnan N, Rao CR (eds) Order statistics: theory \& methods. Handbook of statistics, vol 16. North-Holland, Amsterdam, pp 105-145

Rychlik T (2002) Optimal mean-variance bounds on order statistics from families determined by star ordering. Appl Math (Warsaw) 29:15-32

Rychlik T (2009a) Tight evaluations for expectations of small order statistics from symmetric and symmetric unimodal populations. Stat Probab Lett 79:1488-1493

Rychlik T (2009b) Bounds on expectations of small order statistics from decreasing density populations. Metrika 70:369-381

Rychlik T (2009c) Non-positive upper bounds on expectations of low rank order statistics from DFR populations. Statistics 43:53-63

Shaked M, Shantikumar JG (2007) Stochastic orders. Springer series in statistics. Springer, New York 